\title{
New Classes of Generalized Seminormed Difference Sequence Spaces
}

\author{
M. Mursaleen, ${ }^{1}$ A. Alotaibi, ${ }^{2}$ and Sunil K. Sharma ${ }^{3}$ \\ ${ }^{1}$ Department of Mathematics, Aligarh Muslim University, Aligarh 202002, India \\ ${ }^{2}$ Department of Mathematics, Faculty of Science, King Abdulaziz University, P.O. Box 80203, Jeddah 21589, Saudi Arabia \\ ${ }^{3}$ Department of Mathematics, Model Institute of Engineering \& Technology, Kot Bhalwal 181122, J\&K, India
}

Correspondence should be addressed to M. Mursaleen; mursaleenm@gmail.com

Received 23 March 2014; Revised 2 May 2014; Accepted 4 May 2014; Published 1 June 2014

Academic Editor: Adem Kılıçman

Copyright (C) 2014 M. Mursaleen et al. This is an open access article distributed under the Creative Commons Attribution License, which permits unrestricted use, distribution, and reproduction in any medium, provided the original work is properly cited.

The purpose of this paper is to introduce new classes of generalized seminormed difference sequence spaces defined by a MusielakOrlicz function. We also study some topological properties and prove some inclusion relations between resulting sequence spaces.

\section{Introduction and Preliminaries}

Let $\ell^{0}$ denote the space of all real sequences $x=\left\{x_{k}\right\}$. Let $\mathscr{C}$ denote the space whose elements are the sets of distinct positive integers. Given any element $\sigma$ of $\mathscr{C}$, we denote by $c(\sigma)$ the sequence $\left\{c_{n}(\sigma)\right\}$ such that $c_{n}(\sigma)=1$ if $n \in \sigma$, and $c_{n}(\sigma)=$ 0 otherwise. Further

$$
\mathscr{C}_{s}=\left\{\sigma \in \mathscr{C}: \sum_{n=1}^{\infty} c_{n}(\sigma) \leq s\right\}
$$

the set of those $\sigma$ whose support has cardinality at most $s$, and

$$
\begin{aligned}
& \Phi=\left\{\phi=\left\{\phi_{k}\right\} \in \ell^{0}:\right. \phi_{1}>0, \Delta \phi_{k} \geq 0, \\
&\left.\Delta\left(\frac{\phi_{k}}{k}\right) \leq 0(k=1,2, \ldots)\right\},
\end{aligned}
$$

where $\Delta \phi_{k}=\phi_{k}-\phi_{k-1}$.

For $\phi \in \Phi$, Sargent [1] defined the following sequence space:

$$
m(\phi)=\left\{x=\left\{x_{k}\right\} \in \ell^{0}: \sup _{s \geq 1} \sup _{\sigma \in \mathscr{C}_{s}}\left(\frac{1}{\phi_{s}} \sum_{k \in \sigma}\left|x_{k}\right|\right)<\infty\right\},
$$

The space $m(\phi)$ was extended to $m(\phi, p)$ by Tripathy and Sen [5] as follows:

$$
m(\phi, p)=\left\{x=\left\{x_{k}\right\} \in \ell^{0}: \sup _{s \geq 1} \sup _{\sigma \in \mathscr{C}_{s}}\left(\frac{1}{\phi_{s}} \sum_{k \in \sigma}\left|x_{k}\right|^{p}\right)<\infty\right\} .
$$

The notion of the difference sequence space was introduced by Kizmaz [6] which was generalized by Mursaleen [7]. It was further generalized by Et and Çolak [8] as follows: $Z\left(\Delta^{\mu}\right)=\left\{x=\left(x_{k}\right) \in \omega:\left(\Delta^{\mu} x_{k}\right) \in z\right\}$ for $z=\ell_{\infty}, c$, and $c_{0}$, where $\mu$ is a nonnegative integer and

$$
\Delta^{\mu} x_{k}=\Delta^{\mu-1} x_{k}-\Delta^{\mu-1} x_{k+1}, \quad \Delta^{0} x_{k}=x_{k} \quad \forall k \in \mathbb{N}
$$

or equivalent to the following binomial representation:

$$
\Delta^{\mu} x_{k}=\sum_{v=0}^{\mu}(-1)^{v}\left(\begin{array}{c}
\mu \\
v
\end{array}\right) x_{k+v}
$$

These sequence spaces were generalized by Et and Basarir [9] for $z=\ell_{\infty}(p), c(p)$, and $c_{0}(p)$.

Dutta [10] introduced the following difference sequence spaces using a new difference operator:

$$
Z\left(\Delta_{(\eta)}\right)=\left\{x=\left(x_{k}\right) \in \omega: \Delta_{(\eta)} x \in z\right\} \quad \text { for } z=\ell_{\infty}, c \text {, and } c_{0},
$$

which was further studied in [2-4]. 
where $\Delta_{(\eta)} x=\left(\Delta_{(\eta)} x_{k}\right)=\left(x_{k}-x_{k-\eta}\right)$ for all $k, \eta \in \mathbb{N}$.

In [11], Dutta introduced the sequence spaces $\bar{c}(\|\cdot, \cdot\|$, $\left.\Delta_{(\eta)}^{\mu}, p\right), \bar{c}_{0}\left(\|\cdot\|,, \Delta_{(\eta)}^{\mu}, p\right), \ell_{\infty}\left(\|\cdot, \cdot\|, \Delta_{(\eta)}^{\mu}, p\right), m\left(\|\cdot, \cdot\|, \Delta_{(\eta)}^{\mu}, p\right)$, and $m_{0}\left(\|\cdot, \cdot\|, \Delta_{(\eta)}^{\mu}, p\right)$, where $\eta, \mu \in \mathbb{N}$ and $\Delta_{(\eta)}^{\mu} x_{k}=\left(\Delta_{(\eta)}^{\mu} x_{k}\right)$ $=\left(\Delta_{(\eta)}^{\mu-1} x_{k}-\Delta_{(\eta)}^{\mu-1} x_{k-\eta}\right)$ and $\Delta_{(\eta)}^{0} x_{k}=x_{k}$ for all $k, \eta \in \mathbb{N}$, which is equivalent to the following binomial representation:

$$
\Delta_{(\eta)}^{\mu} x_{k}=\sum_{v=0}^{\mu}(-1)^{v}\left(\begin{array}{c}
\mu \\
v
\end{array}\right) x_{k-\eta v}
$$

The difference sequence spaces have been studied by several authors [12-19] and references therein. Başar and Altay [20] introduced the generalized difference matrix $B=$ $\left(b_{m k}\right)_{k, m \in \mathbb{N}}$ by

$$
b_{m k}= \begin{cases}r, & k=m \\ s, & k=m-1 \\ 0, & (k>m) \text { or }(0 \leq k<m-1) .\end{cases}
$$

Başarir and Kayikçi [21] defined the matrix $B^{\mu}\left(b_{m k}^{\mu}\right)$ which reduces to the difference matrix $\Delta_{(1)}^{\mu}$ if $r=1, s=-1$. The generalized $B^{\mu}$-difference operator is equivalent to the following binomial representation:

$$
B^{\mu} x=B^{\mu}\left(x_{k}\right)=\sum_{v=0}^{\mu}\left(\begin{array}{c}
\mu \\
v
\end{array}\right) r^{\mu-v} s^{v} x_{k-v} .
$$

Let $\wedge=\left(\wedge_{k}\right)$ be a sequence of nonzero scalars. Then, for a sequence space $E$, the multiplier sequence space $E_{\wedge}$, associated with the multiplier sequence $\wedge$, is defined as

$$
E_{\wedge}=\left\{x=\left(x_{k}\right) \in \omega:\left(\wedge_{k} x_{k}\right) \in E\right\} .
$$

Let $\omega(X)$ denote the space of all sequences with elements in $(X, q)$, where $(X, q)$ denotes a seminormed space, seminormed by $q$. The zero sequence is denoted by $\theta=$ $(0,0,0, \ldots)$.

An Orlicz function $M$ is a function, $M:[0, \infty) \rightarrow$ $[0, \infty)$, which is continuous nondecreasing and convex with $M(0)=0, M(x)>0$ for $x>0$ and $M(x) \rightarrow \infty$ as $x \rightarrow \infty$.

Lindenstrauss and Tzafriri [22] used the idea of Orlicz function to define the following sequence space:

$$
\ell_{M}=\left\{x \in \omega: \sum_{k=1}^{\infty} M\left(\frac{\left|x_{k}\right|}{\rho}\right)<\infty\right\}
$$

which is called an Orlicz sequence space. The space $\ell_{M}$ is a Banach space with the norm

$$
\|x\|=\inf \left\{\rho>0: \sum_{k=1}^{\infty} M\left(\frac{\left|x_{k}\right|}{\rho}\right) \leq 1\right\} .
$$

It is shown in [22] that every Orlicz sequence space $\ell_{M}$ contains a subspace isomorphic to $\ell_{p}(p \geq 1)$. The $\Delta_{2^{-}}$ condition is equivalent to $M(L x) \leq K L M(x)$ for all values of $x \geq 0$ and for $L>1$.

A sequence $\mathscr{M}=\left(M_{k}\right)$ of Orlicz functions is called a Musielak-Orlicz function. A sequence $\mathcal{N}=\left(N_{k}\right)$ defined by

$$
N_{k}(v)=\sup \left\{|v| u-M_{k}(u): u \geq 0\right\}, \quad k=1,2, \ldots,
$$

is called the complimentary function of a Musielak-Orlicz function (see $[23,24])$. For a given Musiclak-Orlicz function $\mathscr{M}$, the Musielak-Orlicz sequence space $t_{\mathscr{M}}$ and its subspace $h_{\mathscr{M}}$ are defined as follows:

$$
\begin{gathered}
t_{\mathscr{M}}=\left\{x \in \omega: I_{M}(c x)<\infty \text { for some } c>0\right\}, \\
h_{\mathscr{M}}=\left\{x \in \omega: I_{M}(c x)<\infty \forall c>0\right\},
\end{gathered}
$$

where $I_{\mathscr{M}}$ is a convex modular defined by

$$
I_{\mathscr{M}}(x)=\sum_{k=1}^{\infty} M_{k}\left(x_{k}\right), \quad x=\left(x_{k}\right) \in t_{M} .
$$

We consider $t_{\mathscr{M}}$ equipped with the Luxemburg norm,

$$
\|x\|=\inf \left\{k>0: I_{M}\left(\frac{x}{k}\right) \leq 1\right\},
$$

or equipped with the Orlicz norm,

$$
\|x\|^{0}=\inf \left\{\frac{1}{k}\left(1+I_{M}(k x)\right): k>0\right\} .
$$

A sequence space $E$ is said to be solid if $\left(\alpha_{k} x_{k}\right) \in E$, whenever $\left(x_{k}\right) \in E$ for all sequences $\left(\alpha_{k}\right)$ of scalars such that $\left|\alpha_{k}\right| \leq 1$ for all $k \in \mathbb{N}$.

A sequence space $E$ is said to be monotone if $E$ contains the canonical preimages of all its step spaces.

Remark 1. It is well known that a sequence space is solid implies that it is monotone (see Kamthan and Gupta [25]).

The sequence space $m(\phi)$ was introduced by Sargent [1]. He studied some of its properties and obtained its relationship with the space $\ell_{p}$. Later on, it was investigated from sequence space point of view and related with summability theory by Bilgin [26], Esi [27], Tripathy and Mahanta [28], and many others.

The main goal of the present paper is to introduce new classes of generalized seminormed difference sequence spaces defined by Musielak-Orlicz function.

For a given infinite matrix $A=\left(a_{i k}\right)_{i, k \geq 1}$. The $A$-transform of a sequence $x=\left(x_{k}\right)_{k \geq 1}$ is the sequence $A x=\left(A_{i}\right)(i \geq 1)$, where

$$
A_{i}(x)=\sum_{k=1}^{\infty} a_{i k} x_{k}
$$

provided that the series on the right converges for each $i \geq 1$.

Let $(X, q)$ be a seminormed space, $\mathscr{M}=\left(M_{i}\right)$ a MusielakOrlicz function, and $p=\left(p_{i}\right)$ a bounded sequence of 
positive real numbers. Then we define the following classes of sequences:

$$
\begin{aligned}
& \ell_{\infty}\left(\mathscr{M}, A, B_{\Lambda}^{\mu}, q, p\right) \\
& =\left\{x=\left(x_{k}\right) \in w(X): \sup _{i \geq 1} M_{i}\left(q\left(\frac{A_{i}\left(B_{\Lambda}^{\mu} x_{k}\right)}{\rho}\right)\right)^{p_{i}}<\infty,\right. \\
& \text { for some } \rho>0\} \text {, } \\
& \ell_{1}\left(\mathscr{M}, A, B_{\Lambda}^{\mu}, q, p\right) \\
& =\left\{x=\left(x_{k}\right) \in w(X): \sum_{i=1}^{\infty} M_{i}\left(q\left(\frac{A_{i}\left(B_{\Lambda}^{\mu} x_{k}\right)}{\rho}\right)\right)^{p_{i}}<\infty,\right. \\
& \text { for some } \rho>0\} \text {, } \\
& m\left(\mathscr{M}, A, B_{\Lambda}^{\mu}, \phi, q, p\right) \\
& =\left\{x=\left(x_{k}\right) \in w(X):\right. \\
& \sup _{s \geq 1, \sigma \in \mathscr{C}_{s}} \frac{1}{\phi_{s}} \sum_{i \in \sigma} M_{i}\left(q\left(\frac{A_{i}\left(B_{\Lambda}^{\mu} x_{k}\right)}{\rho}\right)\right)^{p_{i}}<\infty, \\
& \text { for some } \rho>0\} \text {. }
\end{aligned}
$$

The following inequality will be used throughout the paper. If $0<h=\inf p_{k} \leq p_{k} \leq \sup p_{k}=H$ and $D=$ $\max \left(1,2^{H-1}\right)$ then

$$
\left|a_{k}+b_{k}\right|^{p_{k}} \leq D\left\{\left|a_{k}\right|^{p k}+\left|b_{k}\right|^{p_{k}}\right\}
$$

for all $k$ and $a_{k}, b_{k} \in \mathbb{C}$. Also $|a|^{p_{k}} \leq \max \left(1,|a|^{H}\right)$ for all $a \in \mathbb{C}$.

We study here some topological properties and establish inclusion relations between these sequence spaces.

\section{Main Results}

Theorem 2. Let $\mathscr{M}=\left(M_{i}\right)$ be a Musielak-Orlicz function and $p=\left(p_{i}\right)$ a bounded sequence of positive real numbers. Then the spaces $\ell_{\infty}\left(\mathscr{M}, A, B_{\Lambda}^{\mu}, q, p\right), \ell_{1}\left(\mathscr{M}, A, B_{\Lambda}^{\mu}, q, p\right)$, and $m\left(\mathscr{M}, A, B_{\Lambda}^{\mu}, \phi, q, p\right)$ are linear spaces over the field of complex number $\mathbb{C}$.

Proof. Let $x=\left(x_{k}\right), y=\left(y_{k}\right) \in m\left(\mathscr{M}, A, B_{\Lambda}^{\mu}, \phi, q, p\right)$, and $\alpha, \beta \in \mathbb{C}$. Then there exist positive real numbers $\rho_{1}, \rho_{2}>0$ such that

$$
\begin{aligned}
& \sup _{s \geq 1, \sigma \in \mathscr{C}_{s}} \frac{1}{\phi_{s}} \sum_{i \in \sigma} M_{i}\left(q\left(\frac{A_{i}\left(B_{\Lambda}^{\mu} x_{k}\right)}{\rho_{1}}\right)\right)^{p_{i}}<\infty, \\
& \sup _{s \geq 1, \sigma \in \mathscr{C}_{s}} \frac{1}{\phi_{s}} \sum_{i \in \sigma} M_{i}\left(q\left(\frac{A_{i}\left(B_{\Lambda}^{\mu} y_{k}\right)}{\rho_{2}}\right)\right)^{p_{i}}<\infty .
\end{aligned}
$$

Define $\rho_{3}=\max \left(2|\alpha| \rho_{1}, 2|\beta| \rho_{2}\right)$. Since $\left(M_{i}\right)$ is a nondecreasing, convex function and so by using inequality (21), we have

$$
\begin{aligned}
\sum_{i \in \sigma} M_{i}\left(q\left(\frac{A_{i}\left(B_{\Lambda}^{\mu}(\alpha x+\beta y)\right)}{\rho_{3}}\right)\right)^{p_{i}} \\
\leq \sum_{i \in \sigma} M_{i}\left(q\left(\frac{A_{i}\left(B_{\Lambda}^{\mu} \alpha x\right)}{\rho_{3}}\right)+q\left(\frac{A_{i}\left(B_{\Lambda}^{\mu} \beta y\right)}{\rho_{3}}\right)\right)^{p_{i}} \\
\leq D \sum_{i \in \sigma} M_{i}\left(q\left(\frac{A_{i}\left(B_{\Lambda}^{\mu} \alpha x\right)}{\rho_{1}}\right)\right)^{p_{i}} \\
\quad+D \sum_{i \in \sigma} M_{i}\left(q\left(\frac{A_{i}\left(B_{\Lambda}^{\mu} \beta y\right)}{\rho_{2}}\right)\right)^{p_{i}}
\end{aligned}
$$

Thus

$$
\begin{aligned}
\sup _{s \geq 1, \sigma \in \mathscr{C}_{s}} & \frac{1}{\phi_{s}} \sum_{s \in \sigma} M_{i}\left(q\left(\frac{A_{i}\left(B_{\Lambda}^{\mu}(\alpha x+\beta y)\right)}{\rho_{3}}\right)\right)^{p_{i}} \\
\leq & \sup _{s \geq 1, \sigma \in \mathscr{C}_{s}} \frac{1}{\phi_{s}} D \sum_{i \in \sigma} M_{i}\left(q\left(\frac{A_{i}\left(B_{\Lambda}^{\mu} \alpha x\right)}{\rho_{1}}\right)\right)^{p_{i}} \\
& +\sup _{s \geq 1, \sigma \in \mathscr{C}_{s}} \frac{1}{\phi_{s}} D \sum_{i \in \sigma} M_{i}\left(q\left(\frac{A_{i}\left(B_{\Lambda}^{\mu} \beta y\right)}{\rho_{2}}\right)\right)^{p_{i}}<\infty .
\end{aligned}
$$

Thus $(\alpha x+\beta y) \in m\left(\mathscr{M}, A, B_{\Lambda}^{\mu}, \phi, q, p\right)$. Hence $m\left(\mathscr{M}, A, B_{\Lambda}^{\mu}\right.$, $\phi, q, p)$ is a linear space. Similarly, we can prove that the spaces $\ell_{\infty}\left(\mathscr{M}, A, B_{\Lambda}^{\mu}, q, p\right)$ and $\ell_{1}\left(\mathscr{M}, A, B_{\Lambda}^{\mu}, q, p\right)$ are linear spaces. This completes the proof of the theorem.

Theorem 3. Let $\mathscr{M}=\left(M_{i}\right)$ be a Musielak-Orlicz function and $p=\left(p_{i}\right)$ a bounded sequence of positive real numbers. Then $\ell_{1}\left(\mathscr{M}, A, B_{\Lambda}^{\mu}, q, p\right) \subset m\left(\mathscr{M}, A, B_{\Lambda}^{\mu}, \phi, q, p\right) \quad \subset$ $\ell_{\infty}\left(\mathscr{M}, A, B_{\Lambda}^{\mu}, q, p\right)$.

Proof. Let $x=\left(x_{k}\right) \in \ell_{1}\left(\mathscr{M}, A, B_{\Lambda}^{\mu}, q, p\right)$. Then, for some $\rho>$ 0 , we have

$$
\sum_{i=1}^{\infty} M_{i}\left(q\left(\frac{A_{i}\left(B_{\Lambda}^{\mu} x_{k}\right)}{\rho}\right)\right)^{p_{i}}<\infty .
$$

Since $\left(\phi_{n}\right)$ is a monotonic increasing, we have

$$
\begin{gathered}
\frac{1}{\phi_{s}} \sum_{i \in \sigma} M_{i}\left(q\left(\frac{A_{i}\left(B_{\Lambda}^{\mu} x_{k}\right)}{\rho}\right)\right)^{p_{i}} \\
\leq \frac{1}{\phi_{1}} \sum_{i \in \sigma} M_{i}\left(q\left(\frac{A_{i}\left(B_{\Lambda}^{\mu} x_{k}\right)}{\rho}\right)\right)^{p_{i}} \\
\leq \frac{1}{\phi_{1}} \sum_{i=1}^{\infty} M\left(q\left(\frac{A_{i}\left(B_{\Lambda}^{\mu} x_{k}\right)}{\rho}\right)\right)^{p_{i}}<\infty .
\end{gathered}
$$

Hence,

$$
\sup _{s \geq 1, \sigma \in \mathscr{C}_{s}} \frac{1}{\phi_{s}} \sum_{i \in \sigma} M_{i}\left(q\left(\frac{A_{i}\left(B_{\Lambda}^{\mu} x_{k}\right)}{\rho}\right)\right)^{p_{i}}<\infty .
$$


Thus, $x=\left(x_{k}\right) \in m\left(\mathscr{M}, A, B_{\Lambda}^{\mu}, \phi, q, p\right)$. Therefore, $\ell_{1}(\mathscr{M}$, $\left.A, B_{\Lambda}^{\mu}, q, p\right) \subset m\left(\mathscr{M}, A, B_{\Lambda}^{\mu}, \phi, q, p\right)$.

Next, let $x=\left(x_{k}\right) \in m\left(\mathscr{M}, A, B_{\Lambda}^{\mu}, \phi, q, p\right)$. Then, for some $\rho>0$, we have

$$
\sup _{s \geq 1, \sigma \in \mathscr{C}_{s}} \frac{1}{\phi_{s}} \sum_{i \in \sigma} M_{i}\left(q\left(\frac{A_{i}\left(B_{\Lambda}^{\mu} x_{k}\right)}{\rho}\right)\right)^{p_{i}}<\infty .
$$

Hence,

$$
\sup _{s \geq 1, \sigma \in \mathscr{C}_{s}} \frac{1}{\phi_{s}} \sum_{i \in \sigma} M_{i}\left(q\left(\frac{A_{i}\left(B_{\Lambda}^{\mu} x_{k}\right)}{\rho}\right)\right)^{p_{i}}<\infty
$$

(on taking cardinality of $\sigma$ to be 1 ).

Thus, $x=\left(x_{k}\right) \in \ell_{\infty}\left(\mathscr{M}, A, B_{\Lambda}^{\mu}, q, p\right)$. Therefore, $m\left(\mathscr{M}, A, B_{\Lambda}^{\mu}\right.$, $\phi, q, p) \subset \ell_{\infty}\left(\mathscr{M}, A, B_{\Lambda}^{\mu}, q, p\right)$. This completes the proof of the theorem.

Theorem 4. Let $\mathscr{M}=\left(M_{i}\right)$ be a Musielak-Orlicz function and $p=\left(p_{i}\right)$ a bounded sequence of positive real numbers. Then the $\operatorname{spacem}\left(\mathscr{M}, A, B_{\Lambda}^{\mu}, \varphi, q, p\right)$ is a seminormed space, seminormed by

$$
\begin{aligned}
& g(x) \\
& =\inf \left\{\rho>0: \sup _{s \geq 1, \sigma \in \mathscr{C}_{s}} \frac{1}{\phi_{s}} \sum_{i \in \sigma} M_{i}\left(q\left(\frac{A_{i}\left(B_{\Lambda}^{\mu} x\right)}{\rho}\right)\right)^{p_{i}} \leq 1\right\} .
\end{aligned}
$$

Proof. Clearly, $g(x) \geq 0$ for all $x=\left(x_{k}\right) \in$ $m\left(\mathscr{M}, A, B_{\Lambda}^{\mu}, \phi, q, p\right)$ and $g(\theta)=0$. Let $x=\left(x_{k}\right), y=\left(y_{k}\right) \in$ $m\left(\mathscr{M}, A, B_{\Lambda}^{\mu}, \phi, q, p\right)$. Then there exist $\rho_{1}>0$ and $\rho_{2}>0$ such that

$$
\begin{aligned}
& \sup _{s \geq 1, \sigma \in \mathscr{C}_{s}} \frac{1}{\phi_{s}} \sum_{i \in \sigma} M_{i}\left(q\left(\frac{A_{i}\left(B_{\Lambda}^{\mu} x\right)}{\rho_{1}}\right)\right)^{p_{i}} \leq 1, \\
& \sup _{s \geq 1, \sigma \in \mathscr{C}_{s}} \frac{1}{\phi_{s}} \sum_{i \in \sigma} M_{i}\left(q\left(\frac{A_{i}\left(B_{\Lambda}^{\mu} y\right)}{\rho_{2}}\right)\right)^{p_{i}} \leq 1 .
\end{aligned}
$$

Let $\rho=\rho_{1}+\rho_{2}$. Thus, we have

$$
\begin{aligned}
& \sup _{s \geq 1, \sigma \in \mathscr{C}_{s}} \frac{1}{\phi_{s}} \sum_{i \in \sigma} M_{i}\left(q\left(\frac{A_{i}\left(B_{\Lambda}^{\mu}(x+y)\right)}{\rho}\right)\right)^{p_{i}} \\
& =\sup _{s \geq 1, \sigma \in \mathscr{C}_{s}} \frac{1}{\phi_{s}} \sum_{i \in \sigma} M_{i}\left(q\left(\frac{A_{i}\left(B_{\Lambda}^{\mu}(x+y)\right)}{\rho_{1}+\rho_{2}}\right)\right)^{p_{i}} \\
& \leq \sup _{s \geq 1, \sigma \in \mathscr{C}_{s}} \frac{1}{\phi_{s}} \sum_{i \in \sigma}\left\{\frac{\rho_{1}}{\rho_{1}+\rho_{2}} M_{i}\left(q\left(\frac{A_{i}\left(B_{\Lambda}^{\mu}(x)\right)}{\rho_{1}}\right)\right)\right. \\
& \left.\quad+\frac{\rho_{2}}{\rho_{1}+\rho_{2}} M_{i}\left(q\left(\frac{A_{i}\left(B_{\Lambda}^{\mu}(y)\right)}{\rho_{2}}\right)\right)\right\}^{p_{i}} \\
& \leq\left(\frac{\rho_{1}}{\rho_{1}+\rho_{2}}\right) \sup _{s \geq 1, \sigma \in \mathscr{C}_{s}} \frac{1}{\phi_{s}} \sum_{i \in \sigma} M_{i}\left(q\left(\frac{A_{i}\left(B_{\Lambda}^{\mu} x\right)}{\rho_{1}}\right)\right) \\
& +\left(\frac{\rho_{2}}{\rho_{1}+\rho_{2}}\right) \sup _{s \geq 1, \sigma \in \mathscr{C}_{s}} \frac{1}{\phi_{s}} \sum_{i \in \sigma} M_{i}\left(q\left(\frac{A_{i}\left(B_{\Lambda}^{\mu} y\right)}{\rho_{2}}\right)\right)^{p_{i}} \leq 1 .
\end{aligned}
$$

Since the $\rho$ 's are nonnegative, so we have

$$
\begin{aligned}
& g(x+y) \\
& =\inf \left\{\rho>0: \sup _{s \geq 1, \sigma \in \mathscr{C}_{s}} \frac{1}{\phi_{s}} \sum_{i \in \sigma} M_{i}\left(q\left(\frac{A_{i}\left(B_{\Lambda}^{\mu}(x+y)\right)}{\rho}\right)\right)^{p_{i}} \leq 1\right\} \\
& \leq \inf \left\{\rho_{1}>0: \sup _{s \geq 1, \sigma \in \mathscr{C}_{s}} \frac{1}{\phi_{s}} \sum_{i \in \sigma} M_{i}\left(q\left(\frac{A_{i}\left(B_{\Lambda}^{\mu} x\right)}{\rho_{1}}\right)\right)^{p_{i}} \leq 1\right\} \\
& \quad+\inf \left\{\rho_{2}>0: \sup _{s \geq 1, \sigma \in \mathscr{C}_{s}} \frac{1}{\phi_{s}} \sum_{i \in \sigma} M_{i}\left(q\left(\frac{A_{i}\left(B_{\Lambda}^{\mu} y\right)}{\rho_{2}}\right)\right)^{p_{i}} \leq 1\right\} .
\end{aligned}
$$

Thus, $g(x+y) \leq g(x)+g(y)$. Next, for $\lambda \in \mathbb{C}$, without loss of generality, $\lambda \neq 0$, then

$$
\begin{array}{r}
g(\nu x) \\
=\inf \left\{\rho>0: \sup _{s \geq 1, \sigma \in \mathscr{C}_{s}} \frac{1}{\phi_{s}} \sum_{i \in \sigma} M_{i}\left(q\left(\frac{A_{i}\left(B_{\Lambda}^{\mu}(\nu x)\right)}{\rho}\right)\right)^{p_{i}} \leq 1\right\} \\
=\inf \left\{\rho>0: \sup _{s \geq 1, \sigma \in \mathscr{C}_{s}} \frac{1}{\phi_{s}} \sum_{i \in \sigma} M_{i}\left(q\left(\frac{A_{i}\left(B_{\Lambda}^{\mu} x\right)}{r}\right)\right)^{p_{i}} \leq 1\right\}, \\
\text { where } r=\frac{\rho}{|\nu|} .
\end{array}
$$

This completes the proof of the theorem.

Theorem 5. Let $\mathscr{M}=\left(M_{i}\right)$ be a Musielak-Orlicz function and $p=\left(p_{i}\right)$ a bounded sequence of positive real numbers. Then

(i) the space $\ell_{\infty}\left(\mathscr{M}, A, B_{\Lambda}^{\mu}, q, p\right)$ is a seminormed space, seminormed by

$$
f(x)=\inf \left\{\rho>0: \sup _{i \geq 1} M_{i}\left(q\left(\frac{A_{i}\left(B_{\Lambda}^{\mu} x\right)}{\rho}\right)\right)^{p_{i}} \leq 1\right\},
$$

(ii) the space $\ell_{1}\left(\mathscr{M}, A, B_{\Lambda}^{\mu}, q, p\right)$ is a seminormed space, seminormed by

$$
h(x)=\inf \left\{\rho>0: \sum_{i=1}^{\infty} M_{i}\left(q\left(\frac{A_{i}\left(B_{\Lambda}^{\mu} x\right)}{\rho}\right)\right)^{p_{i}} \leq 1\right\} .
$$

Proof. It is easy to prove in view of Theorem 4 , so we omit the details.

Theorem 6. Let $\mathscr{M}=\left(M_{i}\right)$ be a Musielak-Orlicz function and $p=\left(p_{i}\right)$ a bounded sequence of positive real numbers. Then $m\left(\mathscr{M}, A, B_{\Lambda}^{\mu}, \phi, q, p\right) \subset m\left(\mathscr{M}, A, B_{\Lambda}^{\mu}, \psi, q, p\right)$ if and only if $\sup _{s \geq 1}\left(\varphi_{s} / \psi_{s}\right)<\infty$.

Proof. Suppose $\sup _{s \geq 1}\left(\varphi_{s} / \psi_{s}\right)<\infty$ and $x=\left(x_{k}\right) \epsilon$ $m\left(\mathscr{M}, A, B_{\Lambda}^{\mu}, \phi, q, p\right)$. Then, we have for some $\rho>0$

$$
\sup _{s \geq 1, \sigma \in \mathscr{C}_{s}} \frac{1}{\phi_{s}} \sum_{i \in \sigma} M_{i}\left(q\left(\frac{A_{i}\left(B_{\Lambda}^{\mu} x\right)}{\rho}\right)\right)^{p_{i}}<\infty .
$$


Thus,

$$
\begin{aligned}
& \sup _{s \geq 1, \sigma \in \mathscr{C}_{s}} \frac{1}{\psi_{s}} \sum_{i \in \sigma} M_{i}\left(q\left(\frac{A_{i}\left(B_{\Lambda}^{\mu} x\right)}{\rho}\right)\right)^{p_{i}} \\
& \leq\left(\sup _{s \geq 1} \frac{\phi_{s}}{\psi_{s}}\right)\left(\sup _{s \geq 1, \sigma \in \mathscr{C}_{s}} \frac{1}{\phi_{s}} \sum_{i \in \sigma} M_{i}\left(q\left(\frac{A_{i}\left(B_{\Lambda}^{\mu} x\right)}{\rho}\right)\right)^{p_{i}}\right)<\infty .
\end{aligned}
$$

Therefore, $x=\left(x_{k}\right) \in m\left(\mathscr{M}, A, B_{\Lambda}^{\mu}, \psi, q, p\right)$. Hence, $m(\mathscr{M}$, $\left.A, B_{\Lambda}^{\mu}, \phi, q, p\right) \subset m\left(\mathscr{M}, A, B_{\Lambda}^{\mu}, \psi, q, p\right)$.

Conversely, let $m\left(\mathscr{M}, A, B_{\Lambda}^{\mu}, \phi, q, p\right) \subset m\left(\mathscr{M}, A, B_{\Lambda}^{\mu}, \psi\right.$, $q, p)$. Suppose that $\sup _{s \geq 1}\left(\phi_{s} / \psi_{s}\right)=\infty$. Then there exists a sequence of naturals $\left\{s_{i}\right\}$ such that $\lim _{i \rightarrow \infty}\left(\phi_{s_{i}} / \psi_{s_{i}}\right)=\infty$. Let $x=\left(x_{k}\right) \in m\left(\mathscr{M}, A, B_{\Lambda}^{\mu}, \phi, q, p\right)$. Then there exists $\rho>0$ such that

$$
\sup _{s \geq 1, \sigma \in \mathscr{C}_{s}} \frac{1}{\phi_{s}} \sum_{i \in \sigma} M_{i}\left(q\left(\frac{A_{i}\left(B_{\Lambda}^{\mu} x\right)}{\rho}\right)\right)^{p_{i}}<\infty .
$$

Now, we have

$$
\begin{aligned}
& \sup _{s \geq 1, \sigma \in \mathscr{C}_{s}} \frac{1}{\psi_{s}} \sum_{i \in \sigma} M_{i}\left(q\left(\frac{A_{i}\left(B_{\Lambda}^{\mu} x\right)}{\rho}\right)\right)^{p_{i}} \\
& \geq\left(\sup _{s \geq 1} \frac{\phi_{s}}{\psi_{s}}\right)\left(\sup _{s \geq 1, \sigma \in \mathscr{C}_{s}} \frac{1}{\phi_{s}} \sum_{i \in \sigma} M_{i}\left(q\left(\frac{A_{i}\left(B_{\Lambda}^{\mu} x\right)}{\rho}\right)\right)^{p_{i}}\right)=\infty .
\end{aligned}
$$

Therefore, $x=\left(x_{k}\right) \notin m\left(\mathscr{M}, A, B_{\Lambda}^{\mu}, \psi, q, p\right)$, which is a contradiction. Hence $\sup _{s \geq 1}\left(\phi_{s} / \psi_{s}\right)<\infty$.

We get the following corollary as a consequence of Theorem 6 .

Corollary 7. Let $\mathscr{M}=\left(M_{i}\right)$ be a Musielak-Orlicz function and $p=\left(p_{i}\right)$ a bounded sequence of positive real numbers. Then $m\left(\mathscr{M}, A, B_{\Lambda}^{\mu}, \phi, q, p\right)=m\left(\mathscr{M}, A, B_{\Lambda}^{\mu}, \psi, q, p\right)$ if and only if $\sup _{s \geq 1}\left(\phi_{s} / \psi_{s}\right)<\infty$ and $\sup _{s \geq 1}\left(\psi_{s} / \phi_{s}\right)<\infty$ for all $s=$ $1,2,3, \ldots$.

Theorem 8. Let $\mathscr{M}^{\prime}=\left(M_{i}\right)^{\prime}, \mathscr{M}^{\prime \prime}=\left(M_{i}\right)^{\prime \prime}$ be MusielakOrlicz functions which satisfy $\Delta_{2}$-conditions and $p=\left(p_{i}\right)$ a bounded sequence of positive real numbers. Then

(i) $m\left(\mathscr{M}_{\Lambda}^{\prime \mu}, \phi, q, p\right) \subseteq m\left(\mathscr{M}^{\prime} \circ \mathscr{M}_{\Lambda}^{\prime \mu}, A, \phi, q, p\right)$;

(ii) $m\left(\mathscr{M}_{\Lambda}^{\prime \mu}, \phi, q, p\right) \cap m\left(\mathscr{M}_{\Lambda}^{\prime \prime \mu}, \phi, q, p\right) \subseteq m\left(\mathscr{M}^{\prime}+\mathscr{M}_{\Lambda}^{\prime \prime \mu}\right.$, $\phi, q, p)$.

Proof. (i) Let $x=\left(x_{k}\right) \in m\left(\mathscr{M}_{\Lambda}^{\prime \mu}, \phi, q, p\right)$. Then there exists $\rho>0$ such that

$$
\sup _{s \geq 1, \sigma \in \mathscr{C}_{s}} \frac{1}{\phi_{s}} \sum_{i \in \sigma} M_{i}^{\prime}\left(q\left(\frac{A_{i}\left(B_{\Lambda}^{\mu} x\right)}{\rho}\right)\right)^{p_{i}}<\infty .
$$

Let $0<\varepsilon<1$ and $0<\delta<1$ such that $M_{i}(t)<\varepsilon$ for $0 \leq t<\delta$. Let $y_{k}=M_{i}^{\prime}\left(q\left(A_{i}\left(B_{\Lambda}^{\mu} x\right) / \rho\right)\right)^{p_{i}}$ and, for any $\sigma \in \mathscr{C}_{s}$, let $\sum_{i \in \sigma} M_{i}\left(y_{k}\right)=\sum_{1} M_{i}\left(y_{k}\right)+\sum_{2} M_{i}\left(y_{k}\right)$, where the first summation is over $y_{k} \leq \delta$ and the second summation is over $y_{k}>\delta$. Since $\left(M_{i}\right)$ satisfies $\Delta_{2}$-condition, we have

$$
\sum_{1} M_{i}\left(y_{k}\right) \leq M_{i}(1) \sum_{1} y_{k} \leq M_{i}(2) \sum_{1} y_{k} \text {. }
$$

For $y_{k}>\delta$

$$
y_{k}<\frac{y_{k}}{\delta} \leq 1+\frac{y_{k}}{\delta}
$$

Since $\left(M_{i}\right)$ is nondecreasing and convex, so

$$
M\left(y_{k}\right)<M\left(1+\frac{y_{k}}{\delta}\right)<\frac{1}{2} M(2)+\frac{1}{2} M_{i}\left(\frac{2 y_{k}}{\delta}\right) .
$$

Since $\left(M_{i}\right)$ also satisfies $\Delta_{2}$-condition, so

$$
M_{i}\left(y_{k}\right)<\frac{1}{2} K \frac{y_{k}}{\delta} M_{i}(2)+\frac{1}{2} K \frac{y_{k}}{\delta} M_{i}(2)=K \frac{y_{k}}{\delta} M_{i}(2) .
$$

Hence,

$$
\sum_{2} M_{i}\left(y_{k}\right) \leq \max \left(1, K \delta^{-1} M_{i}(2)\right) \sum_{2} y_{k}
$$

By (42) and (46), we have $x=\left(x_{k}\right) \in m\left(\mathscr{M} \circ \mathscr{M}_{\Lambda}^{\prime \mu}, \phi, q, p\right)$. Hence

$$
m\left(\mathscr{M}_{\Lambda}^{\prime \mu}, \phi, q, p\right) \subseteq m\left(\mathscr{M} \circ \mathscr{M}_{\Lambda}^{\prime}, \phi, q, p\right)
$$

(ii) Let $x=\left(x_{k}\right) \in m\left(\mathscr{M}_{\Lambda}^{\prime \mu}, \phi, q, p\right) \cap m\left(\mathscr{M}_{\Lambda}^{\prime \prime \mu}, \phi, q, p\right)$. Then there exists $\rho>0$ such that

$$
\begin{aligned}
& \sup _{s \geq 1, \sigma \in \mathscr{C}_{s}} \frac{1}{\phi_{s}} \sum_{i \in \sigma} M_{i}^{\prime}\left(q\left(\frac{A_{i}\left(B_{\Lambda}^{\mu} x\right)}{\rho}\right)\right)^{p_{i}}<\infty, \\
& \sup _{s \geq 1, \sigma \in \mathscr{C}_{s}} \frac{1}{\phi_{s}} \sum_{i \in \sigma} M_{i}^{\prime \prime}\left(q\left(\frac{A_{i}\left(B_{\Lambda}^{\mu} x\right)}{\rho}\right)\right)^{p_{i}}<\infty .
\end{aligned}
$$

The rest of the proof follows from the equality

$$
\begin{aligned}
& \sum_{i \in \sigma}\left(\mathscr{M}_{i}^{\prime}+\mathscr{M}_{i}^{\prime \prime}\right)\left(q\left(\frac{A_{i}(x)}{\rho}\right)\right)^{p_{i}} \\
& =\sum_{i \in \sigma} M_{i}^{\prime}\left(q\left(\frac{A_{i}\left(B_{\Lambda}^{\mu} x\right)}{\rho}\right)\right)^{p_{i}}+\sum_{i \in \sigma} M_{i}^{\prime \prime}\left(q\left(\frac{A_{i}\left(B_{\Lambda}^{\mu} x\right)}{\rho}\right)\right)^{p_{i}} .
\end{aligned}
$$

This completes the proof of the theorem.

Corollary 9. Let $\mathscr{M}=\left(M_{i}\right)$ be a Musielak-Orlicz function and $p=\left(p_{i}\right)$ a bounded sequence of positive real numbers. Then, we have $m\left(A, B_{\Lambda}^{\mu}, \varphi, q, p\right) \subseteq m\left(\mathscr{M}, A, B_{\Lambda}^{\mu}, \varphi, q, p\right)$.

Proof. It follows from Theorem 8(i) on considering $\mathscr{M}^{\prime}(x)=$ $x$, for all $x \in[0, \infty)$.

The following result is a consequence of Theorem 8 and Corollary 9. 
Corollary 10. Let $\mathscr{M}=\left(M_{i}\right)$ be a Musielak-Orlicz function and $p=\left(p_{i}\right)$ a bounded sequence of positive real numbers. Then $m\left(A, B_{\Lambda}^{\mu}, \varphi, q, p\right) \subseteq m\left(\mathscr{M}, A, B_{\Lambda}^{\mu}, \psi, q, p\right)$ if and only if $\sup _{s \geq 1}\left(\varphi_{s} / \psi_{s}\right)<\infty$.

Theorem 11. Let $\mathscr{M}=\left(M_{i}\right)$ be a Musielak-Orlicz function and $p=\left(p_{i}\right)$ a bounded sequence of positive real numbers. Then the space $m\left(\mathscr{M}, A, B_{\Lambda}^{\mu}, \phi, q, p\right)$ is solid.

Proof. Let $x=\left(x_{k}\right) \in m\left(\mathscr{M}, A, B_{\Lambda}^{\mu}, \phi, q, p\right)$. Then

$$
\sup _{s \geq 1, \sigma \in \mathscr{C}_{s}} \frac{1}{\phi_{s}} \sum_{i \in \sigma} M_{i}\left(q\left(\frac{A_{i}\left(B_{\Lambda}^{\mu} x\right)}{\rho}\right)\right)^{p_{i}}<\infty .
$$

Let $\left(\alpha_{k}\right)$ be a sequence of scalars with $\left|\alpha_{k}\right| \leq 1$ for all $k \in \mathbb{N}$. Then the result follows from (50) and the following inequality

$$
\begin{aligned}
\sum_{i \in \sigma} M_{i}\left(q\left(\frac{A_{i}\left(B_{\Lambda}^{\mu} \alpha x\right)}{\rho}\right)\right)^{p_{i}} & \leq \sum_{i \in \sigma}|\alpha| M_{i}\left(q\left(\frac{A_{i}\left(B_{\Lambda}^{\mu} x\right)}{\rho}\right)\right)^{p_{i}} \\
& \leq \sum_{i \in \sigma} M_{i}\left(q\left(\frac{A_{i}\left(B_{\Lambda}^{\mu} x\right)}{\rho}\right)\right)^{p_{i}} .
\end{aligned}
$$

This completes the proof of the theorem. ies.

In view of the above result, we get the following corollar-

Corollary 12. Let $\mathscr{M}=\left(M_{i}\right)$ be a Musielak-Orlicz function and $p=\left(p_{i}\right)$ a bounded sequence of positive real numbers. Then the space $m\left(\mathscr{M}, A, B_{\Lambda}^{\mu}, \phi, q, p\right)$ is monotone.

We formulate the following result which can be established following the technique of Theorem 11 and Corollary 12.

Corollary 13. Let $\mathscr{M}=\left(M_{i}\right)$ be a Musielak-Orlicz function and $p=\left(p_{i}\right)$ a bounded sequence of positive real numbers. Then the spaces $\ell_{\infty}\left(\mathscr{M}, A, B_{\Lambda}^{\mu}, q, p\right)$ and $\ell_{1}\left(\mathscr{M}, A, B_{\Lambda}^{\mu}, q, p\right)$ are solid and monotone.

Theorem 14. If $(X, q)$ is complete, then $m\left(\mathscr{M}, A, B_{\Lambda}^{\mu}, \phi, q, p\right)$ is also complete.

Proof. Let $\left(x^{j}\right)$ be a Cauchy sequence in $m\left(\mathscr{M}, A, B_{\Lambda}^{\mu}, \phi, q, p\right)$, where $x^{j}=\left(x_{k}^{j}\right)=\left(x_{1}^{j}, x_{2}^{j}, x_{3}^{j}, \ldots\right) \in m\left(\mathscr{M}, A, B_{\Lambda}^{\mu}, \phi, q, p\right)$ for each $j \in \mathbb{N}$. Let $r>0$ and $x_{0}>0$ be fixed. Then for each $\varepsilon / r x_{0}>0$, there exists a positive integer $n_{0}$ such that

$$
g\left(x^{j}-x^{l}\right)<\frac{\varepsilon}{r x_{0}}, \quad \forall j, l \geq n_{0} .
$$

This implies

$$
\begin{aligned}
\inf & \left\{\rho: \sup _{s \geq 1, \sigma \in \mathscr{C}_{s}} \frac{1}{\phi_{s}} \sum_{i \in \sigma} M_{i}\left(q\left(\frac{A_{i}\left(B_{\Lambda}^{\mu}\left(x^{j}-x^{l}\right)\right)}{\rho}\right)\right)^{p_{i}} \leq 1\right\} \\
& <\frac{\varepsilon}{r x_{0}}, \quad \forall j, l \geq n_{0} .
\end{aligned}
$$

We have for all $j, l \geq n_{0}$ and by (53)

$$
\begin{aligned}
& \sup _{s \geq 1, \sigma \in \mathscr{C}_{s}} \frac{1}{\phi_{s}} \sum_{i \in \sigma} M_{i}\left(q\left(\frac{A_{i}\left(B_{\Lambda}^{\mu}\left(x^{j}-x^{l}\right)\right)}{h\left(x^{j}-x^{l}\right)}\right)\right)^{p_{i}} \leq 1 \\
& \Longrightarrow \frac{1}{\phi_{1}} M_{i}\left(q\left(\frac{A_{i}\left(B_{\Lambda}^{\mu}\left(x^{j}-x^{l}\right)\right)}{h\left(x^{j}-x^{l}\right)}\right)\right)^{p_{i}} \leq 1 \\
& \Longrightarrow M_{i}\left(q\left(\frac{A_{i}\left(B_{\Lambda}^{\mu}\left(x^{j}-x^{l}\right)\right)}{h\left(x^{j}-x^{l}\right)}\right)\right)^{p_{i}} \leq \phi_{1}, \quad \forall j, l \geq n_{0} .
\end{aligned}
$$

We can find $r>0$ such that $\left(r x_{0} / 2\right) \eta\left(x_{0} / 2\right)>\phi_{1}$, where $\eta$ is the kernel associated with Musielak-Orlicz function $\mathscr{M}$, such that

$$
\begin{aligned}
& M_{i}\left(q\left(\frac{A_{i}\left(B_{\Lambda}^{\mu}\left(x^{j}-x^{l}\right)\right)}{h\left(x^{j}-x^{l}\right)}\right)\right)^{p_{i}} \leq \frac{r x_{0}}{2} \eta\left(\frac{x_{0}}{2}\right) \\
& \Longrightarrow q\left(A_{i}\left(B_{\Lambda}^{\mu}\left(x^{j}-x^{l}\right)\right)\right)^{p_{i}}<\frac{r x_{0}}{2} \cdot \frac{\varepsilon}{r x_{0}}=\frac{\varepsilon}{2} .
\end{aligned}
$$

Hence $A_{i}\left(B_{\Lambda}^{\mu} x^{j}\right)_{j \geq 1}$ is a Cauchy sequence in $(X, q)$, which is complete. Therefore, for each $k \in \mathbb{N}$, there exist $x_{k} \in X$ and $x=\left(x_{k}\right)$ such that $q\left(A_{i}\left(B_{\Lambda}^{\mu}\left(x^{j}-x\right)\right)\right)^{p_{i}} \rightarrow 0$ as $j \rightarrow \infty$. Using the continuity of $\mathscr{M}$, so for some $\rho>0$, we have

$$
\begin{aligned}
& \sup _{s \geq 1, \sigma \in \mathscr{C}_{s}} \frac{1}{\phi_{s}} \sum_{i \in \sigma} M_{i}\left(q\left(\frac{\lim _{i \rightarrow \infty} A_{i}\left(B_{\Lambda}^{\mu}\left(x^{j}-x^{l}\right)\right)}{\rho}\right)\right)^{p_{i}} \leq 1 \\
& \Longrightarrow \sup _{s \geq 1, \sigma \in \mathscr{C}_{s}} \frac{1}{\phi_{s}} \sum_{i \in \sigma} M_{i}\left(q\left(\frac{A_{i}\left(B_{\Lambda}^{\mu}\left(x^{j}-x^{l}\right)\right)}{\rho}\right)\right)^{p_{i}} \leq 1 .
\end{aligned}
$$

Now, taking the infimum of such $\rho$ 's by (53), we get

$$
\begin{aligned}
& \inf \{\rho>0: \\
&\left.\sup _{s \geq 1, \sigma \in \mathscr{C}_{s}} \frac{1}{\phi_{s}} \sum_{i \in \sigma} M_{i}\left(q\left(\frac{A_{i}\left(B_{\Lambda}^{\mu}\left(x^{j}-x^{l}\right)\right)}{\rho}\right)\right)^{p_{i}} \leq 1\right\}<\varepsilon, \\
& \forall j \geq n_{0} .
\end{aligned}
$$

Since $m\left(\mathscr{M}, A, B_{\Lambda}^{\mu}, \phi, q, p\right)$ is a linear space and $\left(x-x^{j}\right)$ are in $m\left(\mathscr{M}, A, B_{\Lambda}^{\mu}, \phi, q, p\right)$, so it follows that $x=x^{j}+\left(x-x^{j}\right) \epsilon$ $m\left(\mathscr{M}, A, B_{\Lambda}^{\mu}, \phi, q, p\right)$. Hence $m\left(\mathscr{M}, A, B_{\Lambda}^{\mu}, \phi, q, p\right)$ is complete. This completes the proof of the theorem.

\section{Conflict of Interests}

The authors declare that there is no conflict of interests regarding the publication of this paper. 


\section{References}

[1] W. L. C. Sargent, "Some sequence spaces related to the $l^{p}$ spaces," Journal of the London Mathematical Society, vol. 35, pp. 161-171, 1960.

[2] E. Malkowsky and Mursaleen, "Matrix transformations between FK-spaces and the sequence spaces $m(\varphi)$ and $n(\varphi)$," Journal of Mathematical Analysis and Applications, vol. 196, no. 2, pp. 659-665, 1995.

[3] Mursaleen, "Some geometric properties of a sequence space related to $l^{p}$," Bulletin of the Australian Mathematical Society, vol. 67, no. 2, pp. 343-347, 2003.

[4] M. Mursaleen, R. Çolak, and M. Et, "Some geometric inequalities in a new Banach sequence space," Journal of Inequalities and Applications, vol. 2007, Article ID 86757, 6 pages, 2007.

[5] B. C. Tripathy and M. Sen, "On a new class of sequences related to the space $l^{p}$," Tamkang Journal of Mathematics, vol. 33, no. 2, pp. 167-171, 2002.

[6] H. Kızmaz, "On certain sequence spaces," Canadian Mathematical Bulletin, vol. 24, no. 2, pp. 169-176, 1981.

[7] Mursaleen, "Generalized spaces of difference sequences," Journal of Mathematical Analysis and Applications, vol. 203, no. 3, pp. 738-745, 1996.

[8] M. Et and R. Çolak, "On some generalized difference sequence spaces," Soochow Journal of Mathematics, vol. 21, no. 4, pp. 377386, 1995.

[9] M. Et and M. Basarir, "On some new generalized difference sequence spaces," Periodica Mathematica Hungarica, vol. 35, no. 3, pp. 169-175, 1997.

[10] H. Dutta, "On some difference sequence spaces," The Pacific Journal of Science and Technology., vol. 10, pp. 243-247, 2009.

[11] H. Dutta, "Some statistically convergent difference sequence spaces defined over real 2-normed linear spaces," Applied Sciences, vol. 12, pp. 37-47, 2010.

[12] E. Malkowsky, M. Mursaleen, and S. Suantai, "The dual spaces of sets of difference sequences of order $m$ and matrix transformations," Acta Mathematica Sinica, vol. 23, no. 3, pp. 521-532, 2007.

[13] M. Mursaleen, V. Karakaya, H. Polat, and N. Simşek, "Measure of noncompactness of matrix operators on some difference sequence spaces of weighted means," Computers \& Mathematics with Applications, vol. 62, no. 2, pp. 814-820, 2011.

[14] M. Mursaleen and A. K. Noman, "On some new difference sequence spaces of non-absolute type," Mathematical and Computer Modelling, vol. 52, no. 3-4, pp. 603-617, 2010.

[15] M. Mursaleen and A. K. Noman, "Compactness of matrix operators on some new difference sequence spaces," Linear Algebra and its Applications, vol. 436, no. 1, pp. 41-52, 2012.

[16] K. Raj, S. K. Sharma, and A. K. Sharma, "Difference sequence spaces in $n$-normed spaces defined by Musielak-Orlicz function," Armenian Journal of Mathematics, vol. 3, no. 3, pp. 127-141, 2010.

[17] K. Raj and S. K. Sharma, "Some sequence spaces in 2-normed spaces defined by Musielak-Orlicz function," Acta Universitatis Sapientiae. Mathematica, vol. 3, no. 1, pp. 97-109, 2011.

[18] M. Mursaleen, S. K. Sharma, and A. Kılıçman, "Sequence spaces defined by Musielak-Orlicz function over $n$-normed spaces," Abstract and Applied Analysis, vol. 2013, Article ID 364743, 10 pages, 2013.

[19] K. Raj and S. K. Sharma, "Some multiplier sequence spaces defined by a Musielak-Orlicz function in $n$-normed spaces," New Zealand Journal of Mathematics, vol. 42, pp. 45-56, 2012.
[20] F. Başar and B. Altay, "On the space of sequences of $p$ bounded variation and related matrix mappings," Ukrainian Mathematical Journal, vol. 55, no. 1, pp. 108-118, 2003.

[21] M. Başarir and M. Kayikçi, "On the generalized $B^{m}$-Riesz difference sequence space and $\beta$-property," Journal of Inequalities and Applications, vol. 2009, Article ID 385029, 18 pages, 2009.

[22] J. Lindenstrauss and L. Tzafriri, "On Orlicz sequence spaces," Israel Journal of Mathematics, vol. 10, pp. 379-390, 1971.

[23] L. Maligranda, Orlicz Spaces and Interpolation, Seminars in Mathematics, Polish Academy of Science, 1989.

[24] J. Musielak, Orlicz Spaces and Modular Spaces, vol. 1034 of Lecture Notes in Mathematics, Springer, Berlin, Germany, 1983.

[25] P. K. Kamthan and M. Gupta, Sequence Spaces and Series, Marcel Dekker, New York, NY, USA, 1980.

[26] T. Bilgin, "The sequence space $l(p, f, q, s)$ on seminormed spaces," Bulletin of the Calcutta Mathematical Society, vol. 86, no. 4, pp. 295-304, 1994.

[27] A. Esi, "On a class of new type difference sequence spaces related to the space $l^{p}$," Far East Journal of Mathematical Sciences, vol. 13, no. 2, pp. 167-172, 2004.

[28] B. C. Tripathy and S. Mahanta, "On a class of sequences related to the $l^{p}$ space defined by Orlicz functions," Soochow Journal of Mathematics, vol. 29, no. 4, pp. 379-391, 2003. 


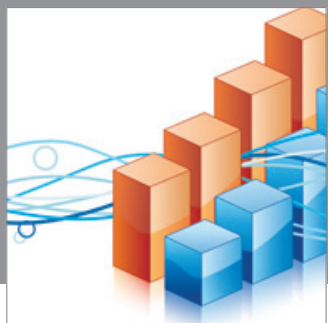

Advances in

Operations Research

mansans

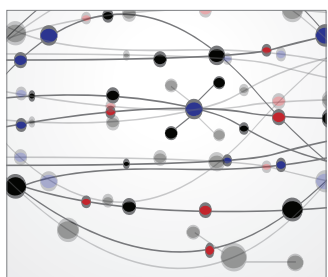

The Scientific World Journal
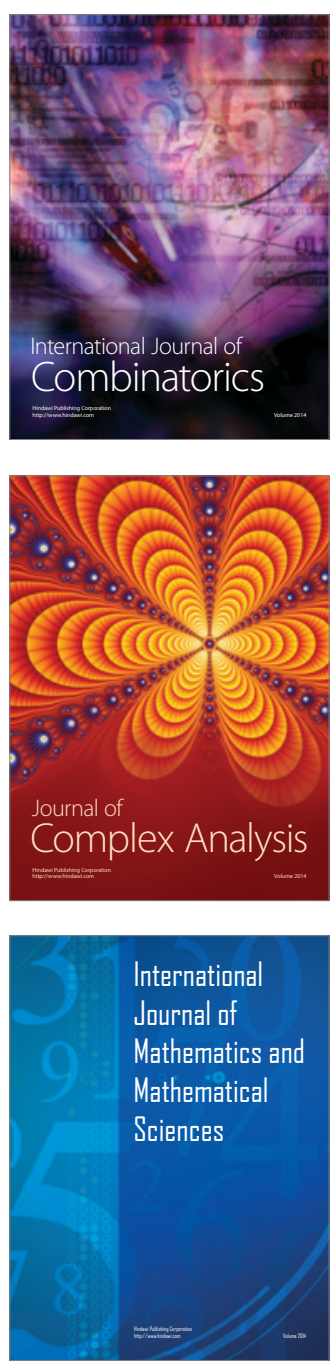
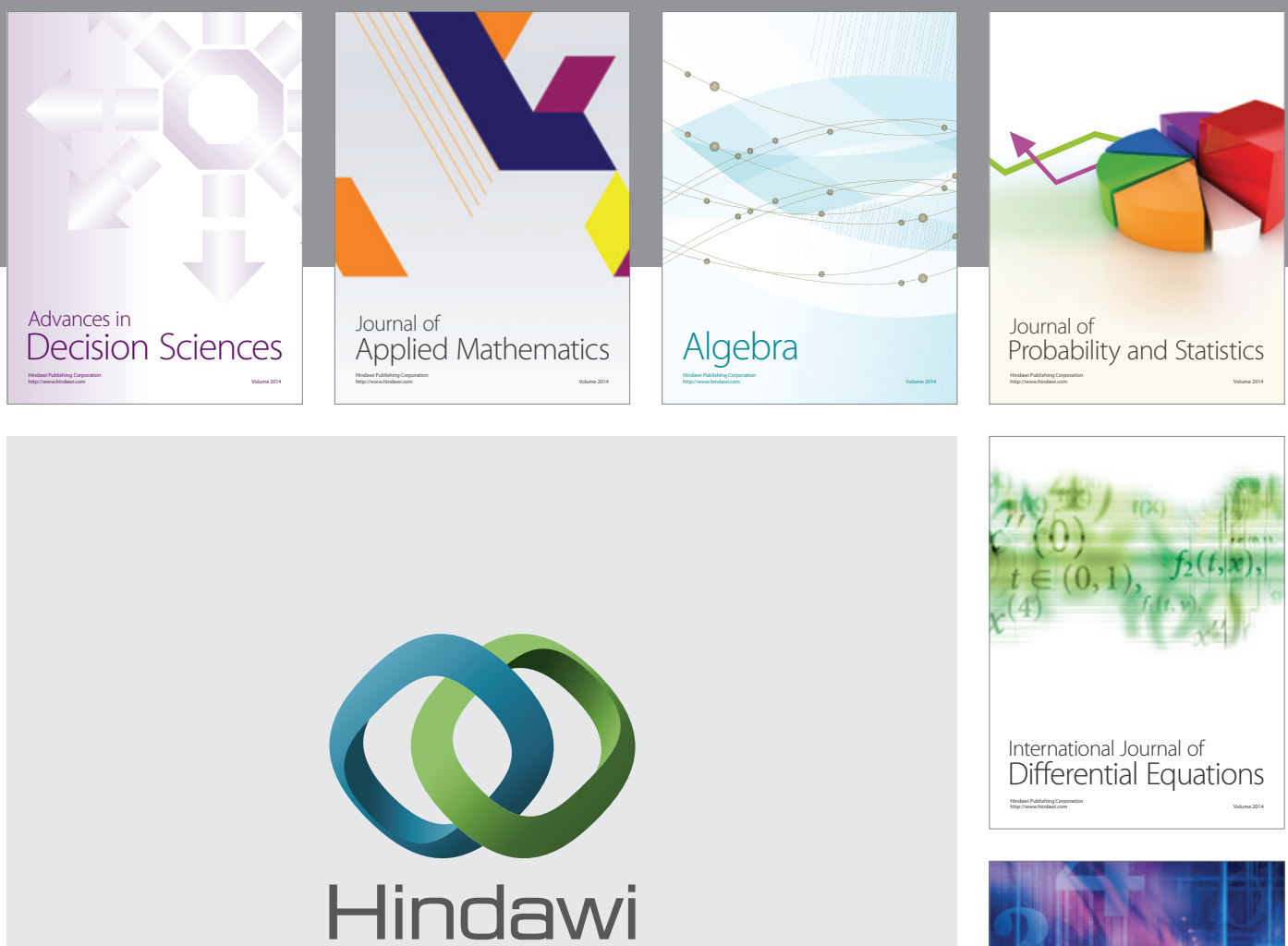

Submit your manuscripts at http://www.hindawi.com
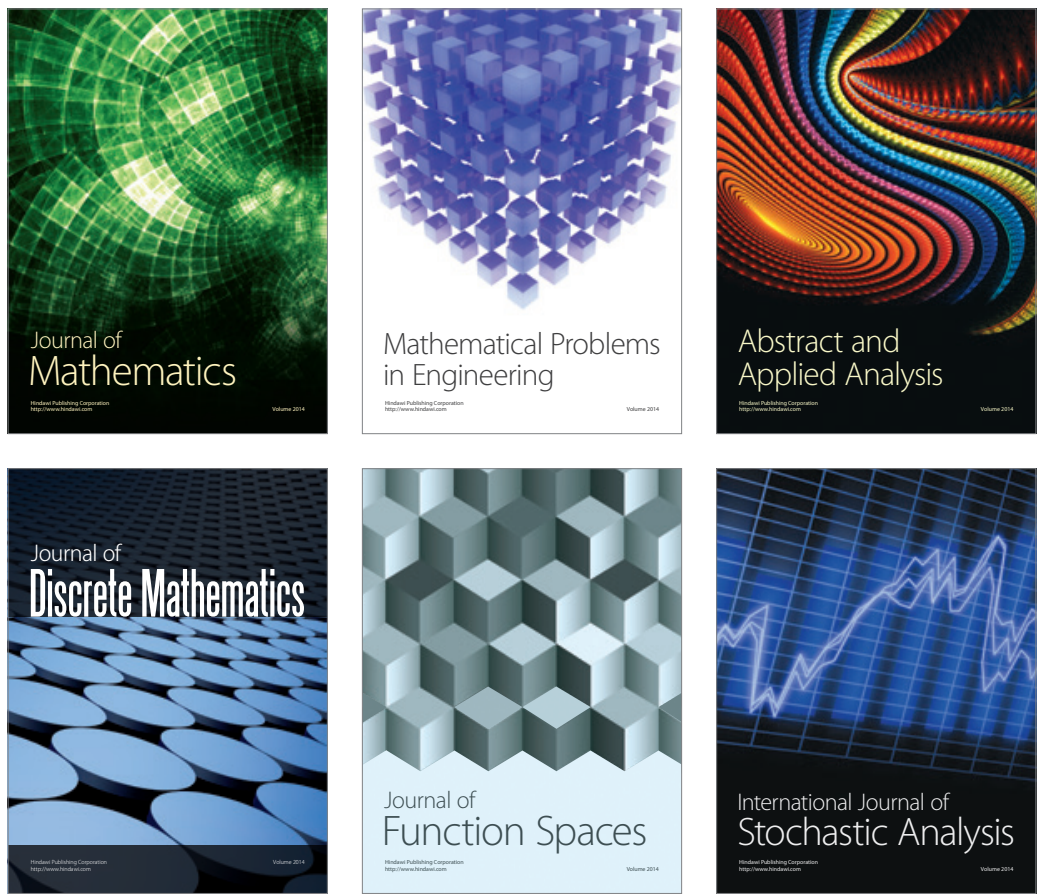

Journal of

Function Spaces

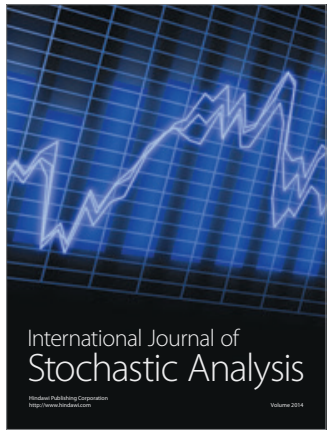

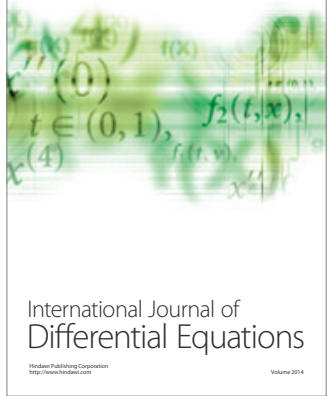
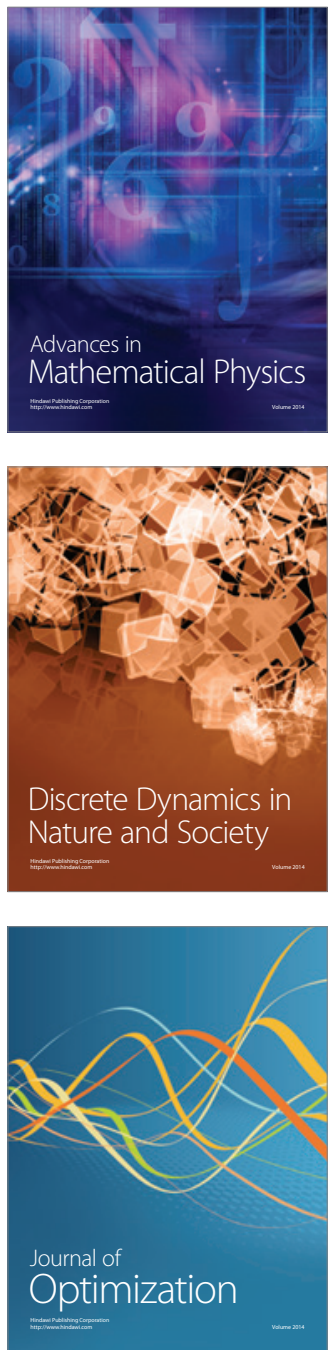